\title{
Hom-Lie triple systems with involution
}

\author{
Amir Baklouti \\ Umm Al-Qura University, College of preliminary year, Department of mathematics, Makkah Al-Mukarramah, Saudi Arabia \\ \& University of Sfax, Faculty of Sciences of Sfax, Soukra 3018 Sfax Pobox, Tunisia
}

Received: 9 December 2018 , Accepted: 7 February 2019

Published online: 29 March 2019.

\begin{abstract}
In this work we we prove that all involutive Hom-Lie triple systems are whether simple or semi-simple. Moreover, we prove that an involutive simple Lie triple system give a rise of Involutive Hom-Lie triple system.
\end{abstract}

Keywords: Jordan triple system, Lie triple system, Casimir operator, quadratic Lie algebra, TKK construction.

\section{Introduction}

The classification of semisimple Lie algebras with involutions can be found in [5]. The Hom-Lie algebras were initially introduced by Hartwig, Larson and Silvestrov in [6] motivated initially by examples of deformed Lie algebras coming from twisted discretizations of vector fields. The Killing form $\mathrm{K}$ of $\mathrm{g}$ is nondegenerate and $\hat{\mathrm{I}}_{\mathrm{s}}$ is symmetric with respect to K. In [1], the author studied Hom-Lie triple system using the double extension and gives an inductive description of quadratic Hom-Lie triple system. In this work we recall the definition of involutive Hom-Lie triple systems and some related structure and we prove that all involutive Hom-Lie triple systems are whether simple or semi-simple. Moreover, we prove that an involutive simple Lie triple system give a rise of Involutive Hom-Lie triple system.

Definition 1. A Hom-Lie triple system is a triple $(\boldsymbol{L},[-,-,-], \alpha)$ consisting of a linear space $\boldsymbol{L}$, a trilinear map $[-,-,-]$ : $\boldsymbol{L} \times \boldsymbol{L} \times \boldsymbol{L} \rightarrow \boldsymbol{L}$ and a linear map $\alpha: \boldsymbol{L} \rightarrow \boldsymbol{L}$ such that

$$
\begin{aligned}
& {[x, x, z]=0, \quad(\text { skewsymmetry) }} \\
& {[x, y, z]+[y, z, x]+[z, x, y]=0, \quad(\text { ternary Jacobi identity) }} \\
& {[\alpha(u), \alpha(v),[x, y, z]]=[[u, v, x], \alpha(y), \alpha(z)]+[\alpha(x),[u, v, y], \alpha(z)]+[\alpha(x), \alpha(y),[u, v, z]],}
\end{aligned}
$$

for all $x, y, z, u, v \in \boldsymbol{L}$. If Moreover $\alpha$ satisfies $\alpha([x, y, z])=[\alpha(x), \alpha(y), \alpha(z)]\left(\right.$ resp. $\left.\alpha^{2}=i d_{\boldsymbol{L}}\right)$ for all $x, y, z \in \boldsymbol{L}$, we say that $(\boldsymbol{L},[-,-,-], \alpha)$ is a multiplicative (resp. involutive) Hom-Lie triple system. A Hom-Lie triple system $(\boldsymbol{L},[-,-,-], \alpha)$ is said to be regular if $\alpha$ is an automorhism of $\boldsymbol{L}$.

When the twisting map $\alpha$ is equal to the identity map, we recover the usual notion of Lie triple system [4,3]. So, Lie triple systems are examples of Hom-Lie triple systems. If we introduce the right multiplication $R$ defined for all $x, y \in \mathbf{L}$ by $R(x, y)(z):=[x, y, z]$, then the conditions above can be written as follow:

$$
\begin{aligned}
& R(x, y)=-R(y, x), \\
& R(x, y) z+R(y, z) x+R(z, x) y=0, \\
& R(\alpha(u), \alpha(v))[x, y, z]=[R(u, v) x, \alpha(y), \alpha(z)]+[\alpha(x), R(u, v) y, \alpha(z)]+[\alpha(x), \alpha(y), R(u, v) z] .
\end{aligned}
$$


We can also introduce the middle (resp. left) multiplication operator $M(x, z) y:=[x, y, z]$ (resp. $L(y, z) x:=[x, y, z])$ for all $x, y, z \in \mathbf{L}$. The equations above can be written in operator form respectively as follows:

$$
\begin{aligned}
& M(x, y)=-L(x, y) \\
& M(x, y)-M(y, x)=R(x, y) \text { for all } x, y \in \mathbf{L} .
\end{aligned}
$$

We can write the equation above as one of the equivalent identities of operators:

$$
\begin{aligned}
& R(\alpha(u), \alpha(v)) R(x, y)-R(\alpha(x), \alpha(y)) R(u, v)=(R(R(u, v) x, \alpha(y))+R(\alpha(x), R(u, v) y)) \alpha . \\
& R(\alpha(u), \alpha(v)) M(x, z)-M(\alpha(x), \alpha(z)) R(u, v)=(M(R(u, v) x, \alpha(z))+M(\alpha(x), R(u, v) z)) \alpha
\end{aligned}
$$

Definition 2. Let $(\boldsymbol{L},[-,-,-], \alpha)$ and $\left(\boldsymbol{L}^{\prime},[-,-,-]^{\prime}, \alpha^{\prime}\right)$ be two two Hom-Lie triple systems. A linear map $f: \boldsymbol{L} \rightarrow \boldsymbol{L}^{\prime}$ is a morphism of Hom-Lie triple systems if

$$
f([x, y, z])=[f(x), f(y), f(z)]^{\prime} \text { and } f \circ \alpha=\alpha^{\prime} \circ f .
$$

In particular, if $f$ is invertible, then $\boldsymbol{L}$ and $\boldsymbol{L}^{\prime}$ are said to be isomorphic.

Definition 3. Let $(\boldsymbol{L},[-,-,-], \alpha)$ be a Hom-Lie triple system and $\boldsymbol{I}$ be a subspace of $\boldsymbol{L}$. We say that $\boldsymbol{I}$ is an ideal of $L$ if $[\boldsymbol{I}, \boldsymbol{L}, \boldsymbol{L}] \subset \boldsymbol{I}$ and $\alpha(\boldsymbol{I}) \subset \boldsymbol{I}$.

Definition 4. A Hom-Lie triple system $\boldsymbol{L}$ is said to be simple (resp. semisimple) if it contains no nontrivial ideal (resp. $\operatorname{Rad}(\boldsymbol{L})=\{0\})$.

According to a result in [2], if $A$ is a Malcev algebra, then $(A,[-,-,-])$ is a Lie triple system with triple product

$$
[x, y, z]=2(x y) z-(z x) y-(y z) x .
$$

Thus, if $A$ is a Malcev algebra and $\alpha: A \rightarrow A$ is an algebra morphism, then, $A_{\alpha}=\left(A,[-,-,-]_{\alpha}=\alpha \circ[-,-,-], \alpha\right)$ is a multiplicative Hom-Lie triple system, where $[-,-,-]$ is the triple product in (3).

Proposition 1. Let $\boldsymbol{L}$ be a Lie triple system and $\alpha$ be an automorphism of $\boldsymbol{L}$. If $\boldsymbol{L}$ is simple, the $\boldsymbol{L}$ is also simple.

Since $\mathbf{L}$ is not abelian, then $\mathbf{L}_{\alpha}$ is also not abelian. Moreover, let $\mathbf{I}$ be an ideal of $\mathbf{L}_{\alpha}$. For all $x, y \in \mathbf{L}$ and $a \in \mathbf{I}$ we have,

$$
[a, x, y]_{\alpha} \in \mathbf{I}
$$

That is,

$$
[\alpha(a), \alpha(x), \alpha(y)] \in \mathbf{I}
$$

Consequently, $\mathbf{I}$ is an ideal of $\mathbf{L}$ because $\alpha$ is an automorphism. Thus, $\mathbf{I}=\{0\}$.

Theorem 1. Let $(\boldsymbol{L},[., .,],. \theta)$ be an involutive Hom-Lie triple system. Then, $\left(\boldsymbol{L},[., ., .]_{\theta}, \theta\right)$ is simple or semi-simple. Moreover, in the second case $\boldsymbol{L}$ can be wrien as $\boldsymbol{L}:=\boldsymbol{L}_{\theta}=\mathscr{S} \oplus \theta(\mathscr{S})$ where $\mathscr{S}$ is a simple ideal of $\boldsymbol{L}$. Conversely, If $(\boldsymbol{L},[., .,],. \theta)$ is an involutive simple Lie triple system, then $\left(\boldsymbol{L},[., ., .]_{\theta}, \boldsymbol{\theta}\right)$ is an involutive Hom-Lie triple system.

Suppose that $\mathbf{L}_{\theta}$ is not simple and put $\mathscr{S}$ a minimal ideal of $\mathbf{L}_{\theta}$. We get $\left[\mathbf{L}_{\theta}, \mathbf{L}_{\theta}, \mathscr{S}\right]_{\theta}$ is an ideal of $\mathbf{L}_{\theta}$ which is contained on $\mathscr{S}$. Thus,

$$
\left[\mathbf{L}_{\theta}, \mathbf{L}_{\theta}, \mathscr{S}\right]_{\theta}=\{0\} \text { or }\left[\mathbf{L}_{\theta}, \mathbf{L}_{\theta}, \mathscr{S}\right]_{\theta}=\mathscr{S} \text {. }
$$

Now, firstly, if $\left[\mathbf{L}_{\theta}, \mathbf{L}_{\theta}, \mathscr{S}\right]_{\theta}=\{0\}$, then $\theta\left(\left[\mathbf{L}_{\theta}\right), \theta\left(\mathbf{L}_{\theta}\right), \theta(\mathscr{S})\right]=\{0\}$. That is, $[\mathbf{L}, \mathbf{L}, \theta(\mathscr{S})]=\{0\}$, because $\theta$ is a bijective linear map. which mean that $\theta(\mathscr{S}) \subset Z(\mathbf{L})=\{0\}$. Thus, $\left[\mathbf{L}_{\theta}, \mathbf{L}_{\theta}, \mathscr{S}\right]_{\theta}=\mathscr{S}$. Hence, $[\mathbf{L}, \mathbf{L}, \theta(\mathscr{S})]=\mathscr{S}$. Which 
implies that $\theta([\mathbf{L}, \mathbf{L}, \theta(\mathscr{S})])=\left[\theta(\mathbf{L}), \theta(\mathbf{L}), \theta^{2}(\mathscr{S})\right]=\theta(\mathscr{S})$. Consequently,

$$
[\mathbf{L}, \mathbf{L}, \mathscr{S}+\theta(\mathscr{S})] \subset \mathscr{S}+\theta(\mathscr{S})
$$

Furthermore,

$$
\theta(\mathscr{S}+\theta(\mathscr{S}))=\theta(\mathscr{S})+\theta^{2}(\mathscr{S})=\theta(\mathscr{S})+\mathscr{S} .
$$

Thus, $\mathscr{S}+\boldsymbol{\theta}(\mathscr{S})$ is an ideal of $(\mathbf{L},[., .,],. \theta)$. Since $\mathscr{S}+\theta(\mathscr{S}) \neq\{0\}$, then $\mathbf{L}=\mathscr{S}+\boldsymbol{\theta}(\mathscr{S})$.

Now, we have to prove that the summation is direct. In fact, since $\theta$ is an automorphism of $\mathbf{L}_{\theta}$, then $\theta(\mathscr{S})$ is an ideal of $\mathbf{L}_{\theta}$. Thus, $\mathscr{S} \cap \theta(\mathscr{S})=\mathscr{S}$ or $\mathscr{S} \cap \theta(\mathscr{S})=\{0\}$ because $\mathscr{S}$ is minimal. Suppose that $\mathscr{S} \cap \theta(\mathscr{S})=\mathscr{S}$, then $\mathscr{S}=\theta(\mathscr{S})$ because $\theta$ is bijective. On the other hand,

$$
[\mathbf{L}, \mathbf{L}, \mathscr{S}]=\theta([\theta(\mathbf{L}), \theta(\mathbf{L}), \theta(\mathscr{S})])=\theta\left([\mathbf{L}, \mathbf{L}, \mathscr{S}]_{\theta}\right) \subset \theta(\mathscr{S})=\mathscr{S}
$$

Thus, $\mathscr{S}$ is an ideal of $(\mathbf{L},[., .,],. \theta)$ and $\mathscr{S}=\mathbf{L}$ because $(\mathbf{L},[., .,]$.$) which contradict the fact that \mathscr{S} \neq \mathbf{L}$ and $\mathscr{S} \neq\{0\}$. Consequently, $\mathscr{S} \cap \boldsymbol{\theta}(\mathscr{S})=\{0\}$ and $\mathbf{L}=\mathscr{S} \oplus \boldsymbol{\theta}(\mathscr{S})$.

Let us prove that $\mathscr{S}$ is a simple ideal of $\left(\mathbf{L}_{\theta},[., ., .]_{\theta}\right)$. In fact, $\mathbf{L}=\mathbf{L}_{\theta}=\mathscr{S} \oplus \theta(\mathscr{S})$. Since $\theta$ is an automorphism of $\mathbf{L}$ then $\theta$ is an automorphism of $\mathbf{L}_{\theta}$.

$$
[\theta(\mathscr{S}), \mathbf{L}, \mathbf{L}]=\theta([\theta(\mathscr{S}), \mathbf{L}, \mathbf{L}]=\theta([\mathscr{S}, \theta(\mathbf{L}), \theta(\mathbf{L})]=\theta([\mathscr{S}, \mathbf{L}, \mathbf{L}]) \subset \theta(\mathscr{S}) .
$$

Thus, $\theta(\mathscr{S})$ is an ideal of $\mathbf{L}_{\theta}$. Furthermore,

$$
[\mathscr{S}, \mathscr{S}, \mathscr{S}]_{\theta}=[\mathscr{S} \oplus \theta(\mathscr{S}), \mathscr{S} \oplus \theta(\mathscr{S}), \mathscr{S}]_{\theta}=\left[\mathbf{L}_{\theta}, \mathbf{L}_{\theta}, \mathscr{S}\right]=\mathscr{S}
$$

Thus, $\mathscr{S}$ is a simple ideal of $\mathbf{L}_{\theta}$ because it is simple with $[\mathscr{S}, \mathscr{S}]_{t} h e t a=\mathscr{S}$. Consequently, $\mathbf{L}_{\theta}$ is semi-simple.

Corollary 1. Let $(\boldsymbol{L},[., .,]$.$) be a Lie triple system with involution \theta$. such that, $\boldsymbol{L}=\mathscr{S} \oplus \boldsymbol{\theta}(\mathscr{S})$ where $\mathscr{S}$ is a simple ideal of $(\boldsymbol{L},[., .,]$.$) . Then the Hom-Lie triple system \left(\boldsymbol{L}_{\theta},[., ., .]_{\theta}, \boldsymbol{\theta}\right)$ is simple.

Let $\mathscr{I}$ be an ideal of $\mathbf{L}_{\theta}$ such that $\mathscr{I} \neq\{0\}$. We have

$$
[\mathbf{L}, \mathbf{L}, \theta(\mathscr{I})]=[\theta(\mathbf{L}), \theta(\mathbf{L}), \theta(\mathscr{I})]=[\mathbf{L}, \mathbf{L}, \mathscr{I}]
$$

because $\mathbf{L}=\theta(\mathbf{L})$ and $\mathscr{I}$ is an ideal of $\mathbf{L}_{\theta}$. Moreover,

$$
[\mathbf{L}, \mathbf{L}, \mathscr{I}]=\theta([\theta(\mathbf{L}), \theta(\mathbf{L}), \theta(\mathscr{I})])=\theta\left([\mathbf{L}, \mathbf{L}, \mathscr{I}]_{t} \text { het } a\right) \subset \theta(\mathscr{I})=\mathscr{I},
$$

because $\mathscr{I}$ is stable under $\theta$ since it is an ideal of the Hom-Lie triple system of $\mathbf{L}_{\theta}$. Consequently, $\mathscr{I}$ is an ideal of $\mathbf{L}$. Thus, $\mathscr{I}=\mathscr{S}$ or $\mathscr{I}=\theta(\mathscr{S})$ or $\mathscr{I}=\mathbf{L}$. Since $\theta(\mathscr{I}) \subset \mathscr{I}$, then $\mathscr{I} \neq \mathscr{S}$ and $\mathscr{I} \neq \theta(\mathscr{S})$. Thus, $\mathscr{I}=\neq \mathscr{S} \oplus \theta(\mathscr{S})=\mathbf{L}$. Moreover, since $[\mathbf{L}, \mathbf{L}, \mathbf{L}]=\mathbf{L}$, then $\left[\mathbf{L}_{\theta}, \mathbf{L}_{\theta}, \mathbf{L}_{\theta}\right]=\mathbf{L}_{\theta}$. Thus, $\left(\mathbf{L}_{\theta},[., .,]_{\theta}, \theta\right)$ is a simple Hom-Lie triple system.

\section{Acknowledgements}

The author would like to thank Deanship of Scientific Research at Umm Al-Qura University (Project ID 15-SCI-3-1-0025) for the financial support. 


\section{Competing interests}

The authors declare that they have no competing interests.

\section{Authors' contributions}

All authors have contributed to all parts of the article. All authors read and approved the final manuscript.

\section{References}

[1] A. Baklouti, Quadratic Hom-Lie triple systems, J. Geom. Phys. Vol. 121, 166-175, (2017).

[2] S. Benayadi, A. Makhlouf, "Hom-Lie algebras with symmetric invariant nondegenerate bilinear forms" : J. Geom. Phys. Vol. 76, 38-60, (2014).

[3] N. Jacobson, Lie and Jordan triple systems, Amer. J. Math., 71, 149-170, (1949).

[4] K. Meyberg, Lectures on algebras and triple systems, Lecture notes, The university of virginia, Charlottesville (1972).

[5] W. G. Lister, A structure theory of Lie triple systems, Trans. Amer. Math. Soc. 72, 217-242, (1952).

[6] J. T. Hartwig, D. Larsson and S. D. Silvestrov, Deformations of Lie algebras using ÏC-derivations. J. Algebra, 295 (2006), $314-361$. 